\title{
Traditional Thai exercise (Ruesi Dadton) for improving motor and cognitive functions in mild cognitive impairment: a randomized controlled trial
}

\author{
Phaksachiphon Khanthong', Kusuma Sriyakul', Ananya Dechakhamphu², Aungkana Krajarng', Chuntida Kamalashiran', \\ Parunkul Tungsukruthai, ${ }^{1, *}$ \\ ${ }^{1}$ Chulabhorn International College of Medicine, Thammasat University, Klonglaung, Thailand \\ ${ }^{2}$ Faculty of Thai Traditional Medicine and Alternative Medicine, Ubon Ratchathani Rajabhat University, Ubon Ratchathani, Thailand
}

This study determined the effectiveness of a 12-week cycle of Ruesi Dadton (RSD) among older adults with mild cognitive impairment (MCI), for improving cognitive and physical performance. Seventy-six participants were included and were divided equally into two groups. A group performed RSD exercise for 60 min, 3 times/wk for 12 weeks, and the control group did not perform RSD exercise. The primary endpoint was cognitive function, as assessed by the Montreal cognitive assessment (MoCA), Mini-Mental State Examination, verbal fluency (VF) test, and trail making test parts $A$ and $B$ (TMT-A and TMT-B). The secondary endpoints were the Timed Up and Go (TUG) test, handgrip, and gait speed results, which were used to evaluate the physical function. There were significant differences in the TMT-B and handgrip scores $(P<0.05)$ between the two groups. Both groups had improved MoCA scores $(P<0.05)$ and normal walking speeds $(P<0.01)$. Additionally, the RSD group showed improved VF test $(P<0.01)$, TMT-B $(P<0.01)$, and TUG test $(P<0.05)$; a negative correlation was found between MoCA and TUG test $(P<0.05)$. However, high walking speed and handgrip $(P<0.05)$ worsened in the control group. RSD exercise resulted in relevant improvements in the cognitive and physical functions in $\mathrm{MCl}$.

Keywords: Cognition, Cognitive dysfunction, Executive function, Exercise, Physical functional performance

\section{INTRODUCTION}

Mild cognitive impairment (MCI) can be indicative of Alzheimer disease (AD) or other forms of dementia. During a follow-up of patients with MCI, progression of MCI to dementia occurred in $29 \%$ of participants, of which $81 \%$ was thought to be caused by underlying AD (Hanfelt et al., 2018). The Montreal cognitive assessment (MoCA) and Mini-Mental State Examination (MMSE) are used to determine the standardised differential diagnosis of MCI (Nasreddine et al., 2005; Petersen et al., 2001). The primary characteristic of $\mathrm{MCI}$ is brain atrophy, leading to cognitive decline, that usually occurs with age but is more than expected; however, the cognitive decline is not as rapid as it is in $\mathrm{AD}$ (Gheysen et al., 2018).
Molecular inflammation leading to functional decline and pathological aging such as that accompanying dementia can be modulated by calorie restriction and exercise (Chung et al., 2009). The main problems in gerontology include physical and cognitive decline that occurs with advancing age (Ma and Chan, 2020). The imbalance in redox systems causes this molecular inflammation that results in multisystemic functional impairment, such as in the musculoskeletal and neurological systems, ultimately leading to disability (El Assar et al., 2020). Accordingly, persons with a low level of physical activity have a corresponding increased rate of memory loss (Nemoto et al., 2018). Moreover, physical inactivity during later life creates a greater risk of dementia (Livingston et al., 2017). The mechanism of physical exercise can promote brain proliferation by evaluating BDNF and irisin (Jin et al., 2018).
${ }^{*}$ Corresponding author: Parunkul Tungsukruthai

(iD https://orcid.org/0000-0002-0536-8002

Chulabhorn International College of Medicine, Thammasat University, Klonglaung, 12120, Pathum Thani, Thailand

Email: parunkul@hotmail.com

Received: September 1, 2021 / Accepted: September 27, 2021
This is an Open Access article distributed under the terms of the Creative Commons Attribution Non-Commercial License (https://creativecommons.org/licenses/by-nc/4.0/) which permits unrestricted non-commercial use, distribution, and reproduction in any medium, provided the original work is properly cited. 
There is strong evidence to suggest that continuous physical activity decreases the risk of dementia (Baumgart et al., 2015). Moreover, exercise is a nonpharmacological and therapeutic approach that can prevent the progression of MCI to dementia (Petersen et al., 2018). Persons with MCI who continue performing physical activity have been found to have improved cognitive function (Gheysen et al., 2018). High level of physical activity is also associated with low risk of MCI and dementia of any type (Laurin et al., 2001). The physical performance not only relates to cognitive function in older adults but is also utilized to predict frailty and sarcopenia such as grip strength, TUG test, and walking speed (Keevil and Romero-Ortuno, 2015). Therefore, individuals with dementia are recommended to exercise during all stages of the disease and in many combinations, to improve their strength, balance, mobility, and ability to perform activities of daily living (ADL) (Blankevoort et al., 2010).

Mind-body exercises are beneficial to persons with MCI (Brenes et al., 2019; Eyre et al., 2017; Sungkarat et al., 2017); however, a recent study found that mind-body intervention had a small effect and relatively low certainty for the cognitive function of individuals with MCI (Demurtas et al., 2020). Hence, further confirmation of such results is needed. Ruesi Dadton (RSD) is a traditional Thai exercise that involves slow movement with deep breathing and breath-holding for well-being particularly in the elderly (Noradechanunt et al., 2017), similar to what is performed in a multimodal exercise program. RSD is also a type of mind-body exercise, just like yoga and Tai Chi. Moreover, a previous study had also reported that multimodal exercises concurrent with high-intensity mental activity can promote physical and cognitive performance (Canli and Ozyurda, 2020). In another study, individuals whose occupation involved working on computers showed significant improvement in motion and a tendency toward improved cognition on performing RSD exercise for 4 days (Tanasugarn et al., 2015). However, there is insufficient evidence regarding the ability of RSD exercise to improve cognitive and physical functions in MCI. We hypothesised that RSD exercise enhances cognitive and physical performance. Therefore, this study aimed to examine the effects of 12 weeks of RSD on cognitive and physical performance in MCI.

\section{MATERIALS AND METHODS}

\section{Study design}

This randomized controlled trial blinded assessors into RSD and control groups. Fig. 1 demonstrates the flow of the study. Ethical

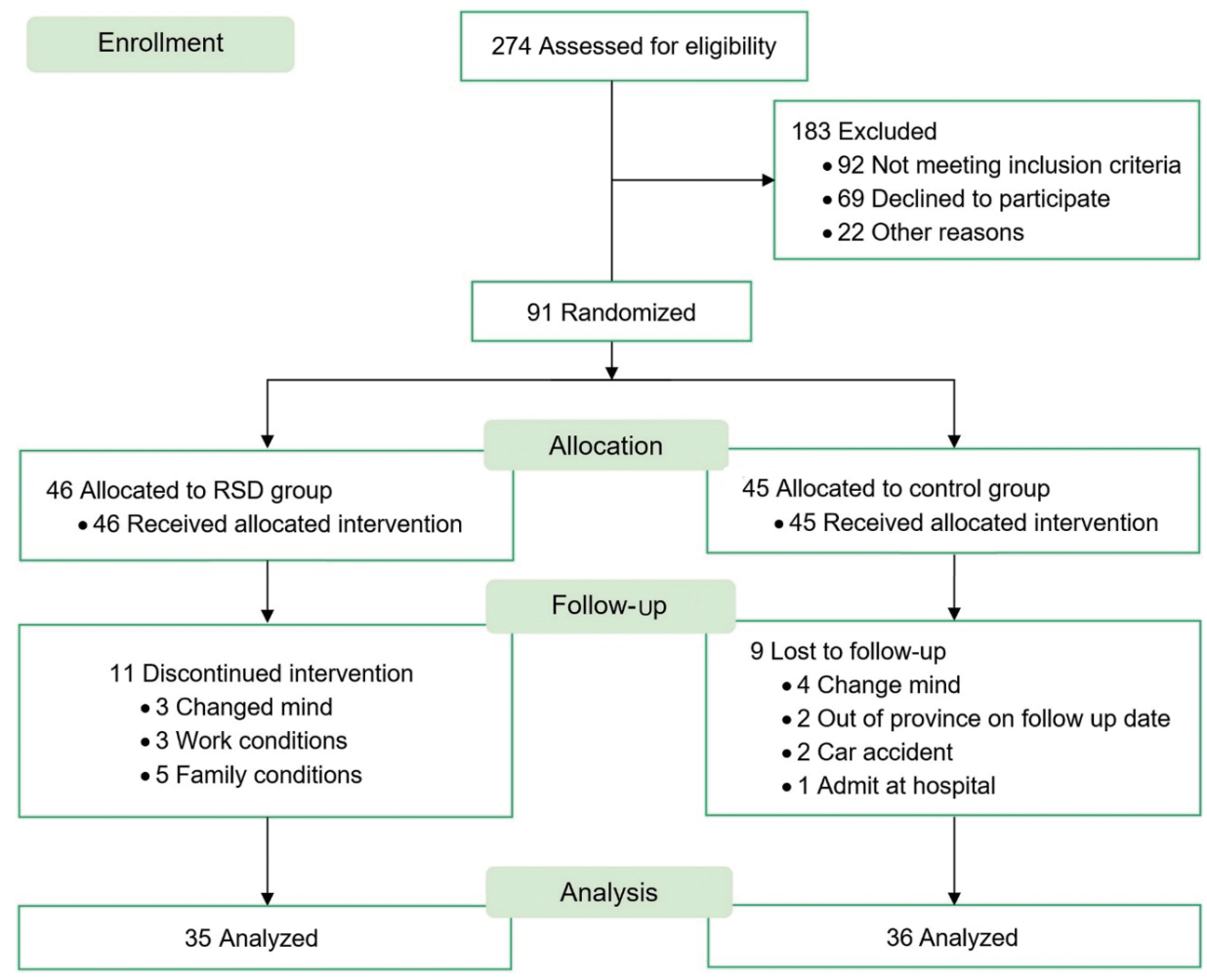

Fig. 1. Consort flow chart. RSD, Ruesi Dadton. 
approval was obtained from The Human Research Ethics Committee No. 1, Faculty of Medicine, Thammasat University, Thailand (no. 119/2562).

\section{Participants}

The participants were recruited at Huadon Health Promoting Hospital, Ubon Ratchathani Province, Thailand, between June and July 2020. A total of 274 individuals aged $50-80$ years were included and grouped according to their ability to read and write (Geda et al., 2010; Northey et al., 2018), hearing ability, speaking ability, ability to communicate in Thai, cognition issues (Petersen, 2004), body mass index (BMI; low to moderate risk, $19-27.5 \mathrm{~kg} / \mathrm{m}^{2}$ ) (WHO Expert Consultation, 2004), MMSE score ( $\geq 24$ points or $<24$ points) (Mitchell et al., 2014), MoCA score ( $<26$ points or $\geq 26$ points) (Nasreddine et al., 2005; Petersen et al., 2001), and diagnosis of amnestic MCI by a neurologist. The exclusion criteria were as follows: dementia; history or diagnosis of cardiovascular disease; neurological disease or mental symptoms; blood pressure $>$ 160/100 mmHg; history of alcoholism or drug addiction; spine problems; history of brain accident or injury; knee pain (visual analogue scale score $\geq 8$ ) or the inability to sit cross-legged; a severe accident during the 3 months before study enrolment; continued regular exercise within the past 2 years ( $\geq 30 \mathrm{~min} /$ day, $\geq 3$ days/wk); and the intent to perform exercise such as RSD, Tai Chi, yoga, or Chi-Qong within 3 months of study enrolment.

\section{Sample size}

A sample size program was used to calculate the number of participants using $\mathrm{T}$ statistics to generate the following two-tailed values: $\alpha=0.05 ; \beta=0.2 ; \mathrm{q} 1=0.5 ; \mathrm{q} 0=0.500 ; \mathrm{E}=0.66$; and $\mathrm{S}=1$ (Huang and Chow, 2019). The standard normal deviation for $\alpha$ was 1.96, the standard normal deviation for $\beta$ was 0.84 , and the standardised effect size (ES) was 0.62 (Kohn and Senyak, 2021). The total number of participants needed was 66; however, an additional $15 \%$ participants were recruited in case of dropout. Therefore, recruitment was complete when 76 participants were enrolled.

\section{Randomization}

A random allocation of participants in each group was operated by randomized block sizes stratified for age and years of formal education. The first criterion for stratification was advanced age because of its association with MCI (Gheysen et al., 2018). The second criterion for stratification was reduced duration of education, which highly contributes to risk of dementia (Livingston et al., 2017).

\section{Blinding}

This trial adopted a single-blinded assessment owing to the nature of experimental protocol; thus, the participants were aware of their group allocation. The data were collected by assessors who were blinded to the group allocation; group numbers were not indicated on participant forms.

\section{Intervention}

Eligible participants were enrolled in the study after screening, and baseline characteristics were recorded. Before the intervention, both groups were provided with information regarding MCI and dementia prevention by the neurologist. The RSD group participated in 2 days of RSD exercise practice; afterward, they started performing RSD exercise using an instruction video for 12 weeks (60 min/session, 3 times/wk). Two Thai medicine instructors led RSD practices for over a period of 2 days; the first day involved breathing exercises and movement corrections, while the second day emphasised deep breathing and breath-holding with slow movements while performing RSD. The researcher (PK) thoroughly supervised the said program. The video included 15 postures with 10 repetitions for each posture according to the performance guidelines of the Thai Ministry of Public Health (Khanthong et al., 2021).

The RSD interventions were conducted after completion of all measurements at baseline. RSD videos, exercise models before a class, supervisor guidance, and 2-4 supervisor assistants were involved in the morning program between 8:00 and 10:00 a.m.

\section{Cognitive tests}

The MoCA and MMSE global cognitive tests were used for screening; individuals with MCI with scores of $\geq 24$ and $<25$ were asked to participate in the study (Nasreddine et al., 2005). The verbal fluency (VF) test score was used to determine memory function; individuals were asked to name as many animals as possible within 1 min (Muangpaisan et al., 2010). The trail making test (TMT) was used to determine executive function: TMT part A (TMT-A) involved drawing a line of numbers from 1 to 25 inside a circle and TMT part B (TMT-B) involved drawing lines from numbers to months inside a circle (in Thai abbreviated language) (Tombaugh, 2004). The results were recorded as seconds using a stopwatch.

\section{Physical tests}

Balance, strength, and speed were measured using the Timed Up and Go (TUG) test, digital hand dynamometer (EH101; Camry, 
Guangdong, China), and walking speed, respectively, and were analyzed to determine physical function. The TUG test was used to evaluate agility and dynamic balance from sitting to standing, walking a distance of $3 \mathrm{~m}$, turning around, and sitting in the same place. To measure handgrip strength, the participants sat face-toface with the physical therapist and flexed their elbows at $90^{\circ}$ with mild wrist extension in a neutral position. The participants were subsequently instructed to squeeze the handle of the dynamometer for $5 \mathrm{sec}$ and, followed by a 2-min rest. Walking speed was measured using a 10-m normal walking speed (NWS) and 5-m high walking speed (HWS) tests conducted by three assessors (an instructor, a timer, and a signal starter).

Table 1. Baseline characteristics of participants who completed posttesting $(\mathrm{N}=71)$

\begin{tabular}{lccc}
\hline Characteristic & RSD $(\mathrm{n}=35)$ & Control $(\mathrm{n}=36)$ & $P$-value \\
\hline Age $(\mathrm{yr})$ & $60.26 \pm 5.67$ & $61.47 \pm 7.49$ & 0.67 \\
Female sex & $31(88.57)$ & $25(69.44)$ & $0.02^{*}$ \\
Primary school & $25(71.43)$ & $21(58.33)$ & 0.40 \\
Weight $(\mathrm{kg})$ & $54.63 \pm 6.58$ & $56.14 \pm 6.67$ & 0.19 \\
BMl $\left(\mathrm{kg} / \mathrm{m}^{2}\right)$ & $23.08 \pm 2.28$ & $23.40 \pm 2.31$ & 0.56 \\
Waist circumference $(\mathrm{cm})$ & $81.87 \pm 7.52$ & $82.89 \pm 5.36$ & 0.86 \\
Agriculture & $23(65.71)$ & $24(66.67)$ & 0.59 \\
Chronic diseases & $15(42.86)$ & $12(33.33)$ & 0.40 \\
MMSE & $26.91 \pm 1.85$ & $26.19 \pm 1.67$ & 0.06 \\
\hline
\end{tabular}

Values are presented as mean \pm standard deviation or number (\%).

RSD, Ruesi Dadton; BMI, body mass index; MMSE, Mini-Mental State Examination. ${ }^{*} P<0.05$, statistically significant differences.

\section{Data analysis}

Data were analyzed using IBM SPSS Statistics ver. 24.0 (IBM Co., Armonk, NY, USA). Descriptive statistics of the frequency, percentage, mean, and standard deviation were used to analyze the data of the RSD and control groups. Comparisons of differences between the groups were performed using chi-square and Fisher exact tests for nominal scales, and the Mann-Whitney $U$-test for interval scales. The McNemar and Wilcoxon signed-rank tests were used to analyze differences within each group before and after the intervention using nominal and interval scales, respectively. The Pearson correlation test was used to determine the relationships of MoCA with cognitive function and physical performance.

\section{RESULTS}

\section{Baseline characteristics}

Seventy-one (RSD group, $n=35$; control group, $n=36$ ) participants were assessed during the analysis. Significant differences were not observed in terms of age, education, BMI, waist circumference, occupation, and illness history of the participants; however, sex was significant $(P<0.05)$ (Table 1). With the exclusion of the dropout participants, this study has an $80.0 \%$ power to detect an ES of 0.665 (Kohn and Senyak, 2021).

\section{Clinical assessments}

MoCA scores were significantly improved in both the RSD and control groups at $P<0.001$ and 0.02 , respectively (Table 2). The RSD group also showed significant improvements at $P<0.01$ for

Table 2. Pre-post assessment

\begin{tabular}{|c|c|c|c|c|c|c|}
\hline \multirow{2}{*}{ Variable } & \multicolumn{3}{|c|}{$\operatorname{RSD}(n=35)$} & \multicolumn{3}{|c|}{ Control $(n=36)$} \\
\hline & Pre & Post & Effect size & Pre & Post & Effect size \\
\hline \multicolumn{7}{|l|}{ Cognition } \\
\hline MoCA & $20.31 \pm 3.31^{\dagger}$ & $22.09 \pm 3.47^{* *}$ & 0.503 & $18.50 \pm 2.92^{\dagger}$ & $19.33 \pm 2.77^{*}$ & 0.367 \\
\hline VF test (n) & $17.03 \pm 3.75$ & $19.51 \pm 4.03^{* *}$ & 0.517 & $16.28 \pm 4.02$ & $17.28 \pm 2.99$ & 0.265 \\
\hline TMT-A (sec) & $61.07 \pm 26.83$ & $58.20 \pm 21.27$ & 0.044 & $64.85 \pm 31.05$ & $62.95 \pm 25.78$ & 0.060 \\
\hline TMT-B (sec) & $174.14 \pm 84.24$ & $133.11 \pm 69.60^{* *}$ & 0.710 & $191.67 \pm 118.07$ & $172.61 \pm 83.88$ & 0.633 \\
\hline \multicolumn{7}{|l|}{ Physical } \\
\hline TUG test (sec) & $10.23 \pm 1.18$ & $9.81 \pm 1.22^{*}$ & 0.381 & $10.57 \pm 1.96$ & $10.58 \pm 1.85$ & 0.033 \\
\hline Handgrip (kg) & $26.08 \pm 5.49$ & $26.18 \pm 5.64$ & 0.006 & $27.35 \pm 7.59$ & $25.71 \pm 7.79^{*}$ & 0.322 \\
\hline 5-m HWS (sec) & $3.65 \pm 0.43$ & $3.72 \pm 0.54$ & 0.103 & $3.68 \pm 0.59$ & $3.81 \pm 0.44^{*}$ & 0.395 \\
\hline 10-m NWS (sec) & $8.88 \pm 0.84$ & $7.88 \pm 0.94^{* *}$ & 0.838 & $8.99 \pm 1.21$ & $7.98 \pm 1.02^{* *}$ & 0.973 \\
\hline
\end{tabular}

Values are presented as mean \pm standard deviation.

RSD, Ruesi Dadton; MoCA, Montreal cognitive assessment; VF, verbal fluency; TMT-A, trail making test part A; TMT-B, trail making test part B; TUG, Timed Up and Go; HWS, high walking speed; NWS, normal walking speed.

${ }^{*} P<0.05$, statistically significant difference within-group. ${ }^{* *} P<0.001$, statistically significant difference within-group. ${ }^{\dagger} P<0.05$, statistically significant difference between-group. 
Table 3. Comparison of change scores

\begin{tabular}{lcccc}
\hline Variable & $\mathrm{RSD}(\mathrm{n}=35)$ & Control $(\mathrm{n}=36)$ & $P$-value & Effect size \\
\hline Cognition & & & & \\
MoCA & $1.77 \pm 2.91$ & $0.83 \pm 2.13$ & 0.16 & 0.189 \\
VF test (n) & $2.49 \pm 3.85$ & $1.00 \pm 4.48$ & 0.18 & 0.182 \\
TMT-A (sec) & $2.86 \pm 24.53$ & $1.61 \pm 21.69$ & 0.95 & 0.009 \\
TMT-B (sec) & $41.09 \pm 57.33$ & $19.06 \pm 99.52$ & $0.02^{*}$ & 0.312 \\
Physical & & & & \\
TUG test (sec) & $0.42 \pm 1.11$ & $-0.01 \pm 1.28$ & 0.25 & 0.157 \\
Handgrip (kg) & $0.10 \pm 2.56$ & $-1.62 \pm 3.83$ & $0.04^{*}$ & 0.283 \\
5-m HWS (sec) & $-0.06 \pm 0.52$ & $-0.14 \pm 0.43$ & 0.33 & 0.133 \\
10-m NWS (sec) & $0.98 \pm 1.00$ & $1.01 \pm 0.90$ & 0.66 & 0.061 \\
\hline
\end{tabular}

Values are presented as mean \pm standard deviation.

RSD, Ruesi Dadton; MoCA, Montreal cognitive assessment; VF, verbal fluency; TMT-A, trail making test part A; TMT-B, trail making test part B; TUG, Timed Up and Go; HWS, high walking speed; NWS, normal walking speed.

${ }^{*} P<0.05$, statistically significant differences.

VF test, TMT-B, and 10-m NWS, and at $P<0.05$ for TUG. The control group showed significant improvement in 10-m NWS scores at $P<0.01$, but the handgrip test and 5-m HWS scores were significantly worse than before $(P<0.05)$.

When the results between the two groups were compared, significant differences in TMT-B $(P=0.02)$ and handgrip $(P=0.04)$ were observed (Table 3). A medium ES was observed in TMT-B, while others exhibited low association.

\section{Correlations among changes in the MoCA scores and physical function}

Changes in the MoCA scores based on the physical function were evaluated (Table 4). Negative correlations between the MoCA and TUG test scores $(r=-0.38, P<0.05)$ were observed in the RSD group.

\section{DISCUSSION}

This study suggests that a 12-week RSD exercise program can induce significant improvements in physical function and reduce cognitive decline. The duration of this study was based on the suggestion of the World Health Organization that older adults ( $\geq 65$ years) should perform at least 150 min of moderate exercise every week (Bull et al., 2020). The physical and cognitive performance results of this study were similar to those of the multimodal exercise program (Canli and Ozyurda, 2020). They were consistent with improved cognitive function observed in individuals aged 50 years who exercised at moderate intensity for 45-60 min per session (Geda et al., 2010; Northey et al., 2018); this also sup-
Table 4. Correlation among changes in the Montreal cognitive assessment scores

\begin{tabular}{lccccc}
\hline \multirow{2}{*}{ Variable } & \multicolumn{2}{c}{$\operatorname{RSD}(\mathrm{n}=35)$} & & \multicolumn{2}{c}{ Control $(\mathrm{n}=36)$} \\
\cline { 2 - 3 } \cline { 5 - 6 } & \multicolumn{1}{c}{$r$} & $P$-value & & $r$ & $P$-value \\
\hline TUG test & -0.38 & $0.02^{*}$ & & 0.25 & 0.13 \\
Handgrip & -0.05 & 0.77 & & -0.06 & 0.72 \\
5-m HWS & -0.14 & 0.41 & & -0.02 & 0.87 \\
10-m NWS & -0.12 & 0.47 & & -0.06 & 0.68 \\
\hline
\end{tabular}

Comparisons were tested using Pearson correlation coefficient analysis. RSD, Ruesi Dadton; TUG, Timed Up and Go; HWS, high walking speed; NWS, normal walking speed.

${ }^{*} P<0.05$, statistically significant differences.

ports the idea that RSD exercise improves the cognitive function of healthy individuals within a short time (Tanasugarn et al., 2015). Participants were allocated to one of two groups based on their risk of MCI determined by age and education level (Luck et al., 2010). Although the baseline characteristics of both groups showed a significant difference in sex, this conflicted with the result of other studies on risk factors for MCI (Baumgart et al., 2015).

For cognition, evaluation of the MoCA scores indicated significant improvements in both groups; this can be attributed to various factors. For example, some MoCA questions (such as subtract 7 from 100) were difficult to answer for a few individuals. Another reason was high sensitivity of the questionnaire, which may have increased the scores (Nasreddine et al., 2005). The TMT-B representing the executive function revealed significant differences between and within groups because higher-complexity tasks require minimal changes to be detectable. Additionally, the results showed no significant difference in RSD, suggesting that TMT-A had simpler tasks than TMT-B. Furthermore, the VF test (representing memory function) scores were significant in the RSD group. These findings indicate that any type of mind-body intervention, such as Tai Chi, can reduce cognitive decline, especially in executive and memory functions of individuals with $\mathrm{MCI}$ and dementia (Gheysen et al., 2018; Nuzum et al., 2020). However, meta-analysis and systematic review of Tai Chi in MCI reported a different impact on cognitive results (Miller and Taylor-Piliae, 2014; Wayne et al., 2014; Wu et al., 2019). Wu et al. (2019) demonstrated that Tai Chi can improve global cognition and memory, but not executive function. Other reviews of Tai Chi found that the executive function can be improved (Miller and Taylor-Piliae, 2014; Wayne et al., 2014). Yoga also resulted in improvements in MCI when compared with the gold standard of memory training and executive function (Eyre et al., 2017).

RSD exercise can induce as much improvement in cognition, 
and executive and memory functions as shown by any other mindbody intervention. The results of the TUG, handgrip strength, and gait speed tests had varying consistency that depended on the duration, movement, posture, and intensity of the mind-body intervention. The TUG test showed significant improvements in the RSD group but not in the control group (Table 2). As a result, the TUG test measures distinct brain activity within the grey matter that is involved with many brain areas and differs between healthy individuals and individuals with MCI (Allali et al., 2020). This correlation may be significant because the task for TUG test is related to the medial temporal lobe, which is the area responsible for cognitive function (Kose et al., 2016). Multimodal exercise has been investigated, but significant improvements were not observed in the exercise group (De Andrade et al., 2013). Although mindbody intervention was a limitation in a number of related studies, significant differences were seen (Hishikawa et al., 2019; Hsieh et al., 2018). Virtual reality-based Tai Chi exercise resulted in many improvements in the physical function, and the control group experienced no significant changes (Hsieh et al., 2018). Individuals with $\mathrm{MCI}$ who practiced yoga and other exercise showed improvements in the TUG test score after 1 year but not within 6 months (Hishikawa et al., 2019).

Significant differences in handgrip strength between individuals with and without decline in cognitive function have been observed. Our data showed a significant decrease in the control group but not in the RSD group, and found a significant difference in strength between the groups. These results were similar to those of a previous study on Tai Chi (Sun et al., 2015) and another study on yoga (Ikai et al., 2017) that found significantly improved handgrip strength. Additional investigations of improvements in strength as a result of mind-body intervention are required.

For gait speed, the 5-m HWS score could possibly may be a more of a sensitive detector than the 10-m NWS score because as no significant decrease in the 5-m HWS score was found in the RSD group and while a significant decrease with a medium ES was detected in the control group. Our results were consistent with those of Sun et al. (2015) wherein the difference between groups after 3 months was not significant. Although there was a slight tendency for decreased 5-m HWS scores, which are consistent with those of a previous study, they were not observed within 3 months; however, they were observed after 6 months of performing Tai Chi (Hsieh et al., 2018; Sun et al., 2015). Therefore, longer follow-up periods are required to confirm the plausibility of these results. In the control group, reducing the speed of cognitive decline, which is a predictor of $\mathrm{MCI}$ and dementia, seemed to reverse dementia
(Grande et al., 2020). Furthermore, another study found that the gait speed of individuals with diabetes could be a predictor and detector of MCI (Machii et al., 2020).

In conclusion, RSD, or traditional Thai exercise, has the potential to improve cognitive function and physical performance and reduce the risk of cognitive decline associated with any type of dementia, especially MCI. To the best of our knowledge, this is the first randomized controlled study on the effects of RSD exercise on individuals with $\mathrm{MCI}$, that also examined cognitive and physical functions.

There were several limitations to this study. First, there was no screening for temporary MCI diagnoses, such as vitamin D and thyroid deficiency testing. Second, we could not limit confounding factors such as ADL and occupational activity performed by both groups. One participant in the control group experienced improvements in all parameters because of changes in behaviour (improved diet and increased exercise) upon being diagnosed with hypertension. Finally, there were limitations to the quality and quantity of mind-body exercise, particularly RSD exercise, performed by individuals with MCI. This study provides preliminary evidence that RSD exercise may be relevant in the prevention of cognitive decline, particularly for complex tasks, in the context of MCI. Furthermore, RSD exercise might lead to improvements in memory, balance, and speed; however, a larger study is required to confirm these results.

\section{CONFLICT OF INTEREST}

No potential conflict of interest to this article was reported.

\section{ACKNOWLEDGMENTS}

The authors wish to thank the Thai Traditional Medical Knowledge Fund and the Thai Ministry of Public Health for funding this study.

\section{REFERENCES}

Allali G, Montembeault M, Griffa A, Beauchet O. Default mode network and the timed up and go in MCI: a structural covariance analysis. Exp Gerontol 2020;129:110748.

Baumgart M, Snyder HM, Carrillo MC, Fazio S, Kim H, Johns H. Summary of the evidence on modifiable risk factors for cognitive decline and dementia: a population-based perspective. Alzheimers Dement 2015; 11:718-726 
Blankevoort CG, van Heuvelen MJ, Boersma F, Luning H, de Jong J, Scherder EJ. Review of effects of physical activity on strength, balance, mobility and ADL performance in elderly subjects with dementia. Dement Geriatr Cogn Disord 2010;30:392-402.

Brenes GA, Sohl S, Wells RE, Befus D, Campos CL, Danhauer SC. The effects of yoga on patients with mild cognitive impairment and dementia: a scoping review. Am J Geriatr Psychiatry 2019;27:188-197.

Bull FC, Al-Ansari SS, Biddle S, Borodulin K, Buman MP, Cardon G, Carty C, Chaput JP, Chastin S, Chou R, Dempsey PC, DiPietro L, Ekelund U, Firth J, Friedenreich CM, Garcia L, Gichu M, Jago R, Katzmarzyk PT, Lambert E, Leitzmann M, Milton K, Ortega FB, Ranasinghe C, Stamatakis E, Tiedemann A, Troiano RP, van der Ploeg HP, Wari V, Willumsen JF. World Health Organization 2020 guidelines on physical activity and sedentary behaviour. Br J Sports Med 2020;54:1451-1462.

Canli S, Ozyurda F. A multi-modal exercise intervention that improves cognitive function and physical performance, elderly with mobilityrelated disability: a randomized controlled trial. J Sports Med Phys Fitness 2020;60:1027-1033.

Chung HY, Cesari M, Anton S, Marzetti E, Giovannini S, Seo AY, Carter C, Yu BP, Leeuwenburgh C. Molecular inflammation: underpinnings of aging and age-related diseases. Ageing Res Rev 2009;8:18-30.

De Andrade LP, Gobbi LT, Coelho FG, Christofoletti G, Costa JL, Stella F. Benefits of multimodal exercise intervention for postural control and frontal cognitive functions in individuals with Alzheimer's disease: a controlled trial. J Am Geriatr Soc 2013;61:1919-1926.

Demurtas J, Schoene D, Torbahn G, Marengoni A, Grande G, Zou L, Petrovic M, Maggi S, Cesari M, Lamb S, Soysal P, Kemmler W, Sieber C, Mueller C, Shenkin SD, Schwingshackl L, Smith L PhD, Veronese N; European Society of Geriatric Medicine Special Interest Group in Systematic Reviews and Meta-Analyses, Frailty, Sarcopenia, and Dementia. Physical activity and exercise in mild cognitive impairment and dementia: an umbrella review of intervention and observational studies. J Am Med Dir Assoc 2020;21:1415-1422.e6

El Assar M, Angulo J, Rodríguez-Mañas L. Frailty as a phenotypic manifestation of underlying oxidative stress. Free Radic Biol Med 2020;149: 72-77.

Eyre HA, Siddarth P, Acevedo B, Van Dyk K, Paholpak P, Ercoli L, St Cyr $\mathrm{N}$, Yang $\mathrm{H}$, Khalsa DS, Lavretsky $\mathrm{H}$. A randomized controlled trial of Kundalini yoga in mild cognitive impairment. Int Psychogeriatr 2017; 29:557-567.

Geda YE, Roberts RO, Knopman DS, Christianson TJ, Pankratz VS, Ivnik RJ, Boeve BF, Tangalos EG, Petersen RC, Rocca WA. Physical exercise, aging, and mild cognitive impairment: a population-based study. Arch Neurol 2010;67:80-86.

Gheysen F, Poppe L, DeSmet A, Swinnen S, Cardon G, De Bourdeaudhuij
I, Chastin S, Fias W. Physical activity to improve cognition in older adults: can physical activity programs enriched with cognitive challenges enhance the effects? A systematic review and meta-analysis. Int J Behav Nutr Phys Act 2018;15:63.

Grande G, Rizzuto D, Vetrano DL, Marseglia A, Vanacore N, Laukka EJ, Welmer AK, Fratiglioni L. Cognitive and physical markers of prodromal dementia: a 12-year-long population study. Alzheimers Dement 2020;16:153-161.

Hanfelt JJ, Peng L, Goldstein FC, Lah JJ. Latent classes of mild cognitive impairment are associated with clinical outcomes and neuropathology: Analysis of data from the National Alzheimer's Coordinating Center. Neurobiol Dis 2018;117:62-71.

Hishikawa N, Takahashi Y, Fukui Y, Tokuchi R, Furusawa J, Takemoto M, Sato K, Yamashita T, Ohta Y, Abe K. Yoga-plus exercise mix promotes cognitive, affective, and physical functions in elderly people. Neurol Res 2019;41:1001-1007.

Hsieh CC, Lin PS, Hsu WC, Wang JS, Huang YC, Lim AY, Hsu YC. The effectiveness of a virtual reality-based Tai Chi exercise on cognitive and physical function in older adults with cognitive impairment. Dement Geriatr Cogn Disord 2018;46:358-370.

Huang Z, Chow SC. Probability monitoring procedures for sample size determination. J Biopharm Stat 2019;29:887-896.

Ikai S, Uchida H, Mizuno Y, Tani H, Nagaoka M, Tsunoda K, Mimura M, Suzuki T. Effects of chair yoga therapy on physical fitness in patients with psychiatric disorders: a 12-week single-blind randomized controlled trial. J Psychiatr Res 2017;94:194-201.

Jin Y, Sumsuzzman DM, Choi J, Kang H, Lee SR, Hong Y. Molecular and functional interaction of the myokine irisin with physical exercise and Alzheimer's disease. Molecules 2018;23:3229.

Keevil VL, Romero-Ortuno R. Ageing well: a review of sarcopenia and frailty. Proc Nutr Soc 2015;74:337-347.

Kohn MA, Senyak J. Sample size calculators [Internet]. San Francisco: UCSF CTSI; 2021 [cited 2021 June 26]. Available from: https://www. sample-size.net/.

Khanthong P, Natason A, Dechakhamphu A. Benefit of Ruesi Dadton on oxidative stress and physical performance: quasi-experimental study. Phys Occup Ther Geriatr 2021:1-15. https://doi.org/10.1080/02703181.2 021.1980480.

Kose Y, Ikenaga M, Yamada Y, Morimura K, Takeda N, Ouma S, Tsuboi Y, Yamada T, Kimura M, Kiyonaga A, Higaki Y, Tanaka H; Nakagawa Study Group. Timed Up and Go test, atrophy of medial temporal areas and cognitive functions in community-dwelling older adults with normal cognition and mild cognitive impairment. Exp Gerontol 2016; 85:81-87.

Laurin D, Verreault R, Lindsay J, MacPherson K, Rockwood K. Physical 
activity and risk of cognitive impairment and dementia in elderly persons. Arch Neurol 2001;58:498-504.

Livingston G, Sommerlad A, Orgeta V, Costafreda SG, Huntley J, Ames

D, Ballard C, Banerjee S, Burns A, Cohen-Mansfield J, Cooper C, Fox

N, Gitlin LN, Howard R, Kales HC, Larson EB, Ritchie K, Rockwood

K, Sampson EL, Samus Q, Schneider LS, Selbæk G, Teri L, Mukadam

N. Dementia prevention, intervention, and care. Lancet 2017;390:26732734.

Luck T, Luppa M, Briel S, Riedel-Heller SG. Incidence of mild cognitive impairment: a systematic review. Dement Geriatr Cogn Disord 2010; 29:164-175.

Ma L, Chan P. Understanding the physiological links between physical frailty and cognitive decline. Aging Dis 2020;11:405-418.

Machii N, Kudo A, Saito H, Tanabe H, Iwasaki M, Hirai H, Masuzaki H, Shimabukuro M. Walking speed is the sole determinant criterion of sarcopenia of mild cognitive impairment in Japanese elderly patients with type 2 diabetes mellitus. J Clin Med 2020;9:2133.

Miller SM, Taylor-Piliae RE. Effects of Tai Chi on cognitive function in community-dwelling older adults: a review. Geriatr Nurs 2014;35:9-19.

Mitchell AJ, Shukla D, Ajumal HA, Stubbs B, Tahir TA. The Mini-Mental State Examination as a diagnostic and screening test for delirium: systematic review and meta-analysis. Gen Hosp Psychiatry 2014;36:627633.

Muangpaisan W, Intalapaporn S, Assantachai P. Digit span and verbal fluency tests in patients with mild cognitive impairment and normal subjects in Thai-community. J Med Assoc Thai 2010;93:224-230.

Nasreddine ZS, Phillips NA, Bédirian V, Charbonneau S, Whitehead V, Collin I, Cummings JL, Chertkow H. The Montreal Cognitive Assessment, MoCA: a brief screening tool for mild cognitive impairment. J Am Geriatr Soc 2005;53:695-699.

Nemoto Y, Sato S, Takahashi M, Takeda N, Matsushita M, Kitabatake Y, Maruo K, Arao T. The association of single and combined factors of sedentary behavior and physical activity with subjective cognitive complaints among community-dwelling older adults: cross-sectional study. PLoS One 2018;13:e195384.

Noradechanunt C, Worsley A, Groeller H. Thai Yoga improves physical function and well-being in older adults: a randomised controlled trial. J Sci Med Sport 2017;20:494-501.

Northey JM, Cherbuin N, Pumpa KL, Smee DJ, Rattray B. Exercise interventions for cognitive function in adults older than 50: a systematic review with meta-analysis. Br J Sports Med 2018;52:154-160.
Nuzum H, Stickel A, Corona M, Zeller M, Melrose RJ, Wilkins SS. Potential benefits of physical activity in $\mathrm{MCI}$ and dementia. Behav Neurol 2020;2020:7807856.

Petersen RC. Mild cognitive impairment as a diagnostic entity. J Intern Med 2004;256:183-194.

Petersen RC, Lopez O, Armstrong MJ, Getchius TSD, Ganguli M, Gloss D, Gronseth GS, Marson D, Pringsheim T, Day GS, Sager M, Stevens J, Rae-Grant A. Practice guideline update summary: mild cognitive impairment: report of the Guideline Development, Dissemination, and Implementation Subcommittee of the American Academy of Neurology. Neurology 2018;90:126-135.

Petersen RC, Stevens JC, Ganguli M, Tangalos EG, Cummings JL, DeKosky ST. Practice parameter: early detection of dementia: mild cognitive impairment (an evidence-based review). Report of the Quality Standards Subcommittee of the American Academy of Neurology. Neurology 2001;56:1133-1142.

Sun J, Kanagawa K, Sasaki J, Ooki S, Xu H, Wang L. Tai chi improves cognitive and physical function in the elderly: a randomized controlled trial. J Phys Ther Sci 2015;27:1467-1471.

Sungkarat S, Boripuntakul S, Chattipakorn N, Watcharasaksilp K, Lord SR. Effects of Tai Chi on cognition and fall risk in older adults with mild cognitive impairment: a randomized controlled trial. J Am Geriatr Soc 2017;65:721-727.

Tanasugarn L, Natearpha P, Kongsakon R, Chaosaowapa M, Choatwongwachira W, Seanglaw D, Kiratisin P, Namatra C, Srinonprasert V, Nimmannnit A, Vannabhum M, Laohapand T, Kuptniratsaikul V. Physical effects and cognitive function after exercising "Rue-si-dadton" (exercise using the posture of the hermit doing body contortion): a randomized controlled pilot trial. J Med Assoc Thai 2015;98:306-313.

Tombaugh TN. Trail Making Test A and B: normative data stratified by age and education. Arch Clin Neuropsychol 2004;19:203-214.

Wayne PM, Walsh JN, Taylor-Piliae RE, Wells RE, Papp KV, Donovan NJ, Yeh GY. Effect of tai chi on cognitive performance in older adults: systematic review and meta-analysis. J Am Geriatr Soc 2014;62:25-39.

WHO Expert Consultation. Appropriate body-mass index for Asian populations and its implications for policy and intervention strategies. Lancet 2004;363:157-163.

Wu C, Yi Q, Zheng X, Cui S, Chen B, Lu L, Tang C. Effects of mind-body exercises on cognitive function in older adults: a meta-analysis. J Am Geriatr Soc 2019;67:749-758. 